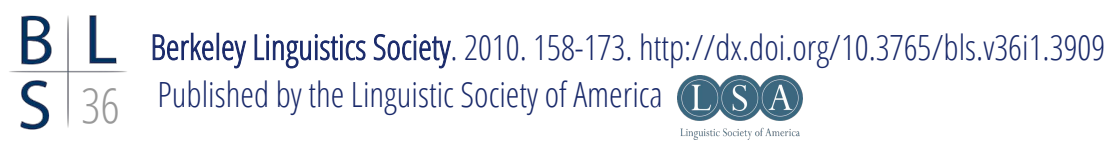

\title{
On the Reflexive-Antipassive Polysemy: Typological Convergence from Unrelated Languages
}

\author{
KATARZYNA JANIC \\ Université Lumière Lyon 2
}

\section{$1 \quad$ Introduction}

This paper deals with the antipassive construction that has raised considerable interest in the syntactic description of ergative languages over the last few decades (Dixon 1979). Often defined on structural grounds, antipassive is said to be a derived intransitive construction with a two-place predicate, the object argument of which is either suppressed or realized as an oblique (Polinsky 2005). Traditionally, the antipassive is related to the ergative system. Although some authors insist on the link between the antipassive and ergativity (Dixon 1979, Cooreman 1993), certain typologically-oriented publications extend discussions on antipassive phenomena to accusative languages (Heath 1976, Polinsky 2005, Creissels 2006).

To derive the antipassive construction, ergative languages use an antipassive marker that may have a different impact on the clause. It can either affect its syntax, making the transitive clause intransitive, or additionally it can also affect the semantics of the verb. Indeed, the syncretism of antipassive markers with aspect/modality categories is frequently observed across different ergative languages.

Apart from its correspondence to aspect/modality categories, the antipassive marker can also be related to the reflexive/middle function such as in Australian languages. A typological investigation in the development of the reflexive marker shows that the same polysemy also exists in accusative languages, in particular in Slavic (Nedjalkov 2007). In addition to Australian and Slavic languages, Romance, Cariban, Tacanan, Manding, South Caucasian, and Chukotko-Kamchatkan languages can be mentioned among language families in which the reflexiveantipassive polysemy is attested. The fact that the same pattern is observed in genetically unrelated languages provides clear evidence that the morphological coincidence is by no means homophonous in nature.

Up to now, research into reflexive-antipassive polysemy has received relative- 
ly little attention. Apart from a few attempts (Terrill 1997, Polinsky 2005, Nedjalkov 2006, Creissels 2006), the question of the reflexive-antipassive polysemy has not been investigated extensively. This paper extends on the existing work with two objectives. First, it argues in favor of the recognition of antipassives in accusative languages. Second, it aims to show that the shared morphology of reflexives and antipassives is by no means accidental but historically grounded.

To argue for the historical account of the reflexive-to-antipassive extension, I will follow Terrill (cf. 1997 for Australian languages) and I will suggest that antipassives developed from reflexivity through functional extension. Contrary to Terrill (1997), however, I will not restrict my analysis to a single language family. The intention of this paper is to investigate a range of unrelated language families to show that the historical explanation of the shared morphology is legitimate, regardless of the language system. The account of the given polysemous pattern will be based on the functional approach proposed by Givón (2001). Building on his analysis, I will show that contrary to Australian languages in which the reflexive-to-antipassive extension is in its late stage of grammaticalization, in all other languages, the antipassive is still in its early stage of development, characterized by functional ambiguity.

\section{Reflexive-Antipassive Polysemy}

Within the scope of meanings subsumed under the reflexive marker, some of them are directly related to reflexivity, whereas some others have developed from it in an indirect way. Regardless of their direct or non-direct relatedness to the reflexive notion, the fact that the reflexive morpheme developed a number of different meanings ordered on the evolutionary scale remains unquestionable. Consequently, typologically-oriented studies on the polysemy of the reflexive marker tend not only to establish a number of possible meanings the reflexive morpheme can express, but also to define to what extent they are related to each other. This raises a further question of the semantic interrelations between the meanings and their degree of productivity. Indeed, whereas certain meanings are very common and productive, some others remain rare, represented by a limited number of verbs. Still, their existence is significant for the typological study, provided they occur across different languages.

This paper concerns languages with the unproductive type of polysemy, i.e. when the respective marker apart from the reflexive function, expresses also the non-reflexive meaning, i.e. the antipassive one. The following sections investigate in detail a number of languages in which the reflexive-antipassive polysemy is attested. Due to lack of space, I will limit myself to a few language families only. 
On the Reflexive - Antipassive Polysemy

\subsection{Reflexive-Antipassive Polysemy in the Slavic Family}

The Slavic languages are well-known for the polysemous property of their reflexive marker. The following two examples illustrate the reflexive and antipassive derivation in Bulgarian respectively:

(1a) Toj porjaza prǔsta

he cut.PST.3SG finger

'He cut his finger.'

(1b) Toj se porjaza

he REFL cut.PST.3SG

'He cut himself = his finger.'

(Geniušienè 1988:247)

(2a) Toj buta vsički

he push.PRS.3SG everybody

'He pushes everybody.'

(2b) Toj se buta

he AP push.PRS.3SG

'He pushes [everybody].'

*'He pushes himself.'

(Geniušienè 1988:249)

In both examples the presence of the se morpheme triggers an obligatory deletion of the object argument. In (1), however, the removed constituent acquires an anaphoric interpretation, whereas in (2), it functions as a non-referring object of the antipassive.

The antipassive meaning of a reflexive marker may also be expressed on the same verbal stem; cf. (3) where the Polish reflexive form drapie sie has two meanings. In this case we deal with the reflexive-antipassive polysemy of a given derivative.

(3) Proszę pani, a on się drapie

Excuse me Madam but he AP scratch.PRS.3SG

'Excuse me Madam, but he is scratching himself.' (reference to a child sick with smallpox)

'Madam, he is scratching [other children].'

In (3), the semantic overlap between the reflexive and antipassive meaning pertains primarily to the lexical meaning of the base verb. The verb 'scratch' denotes a type of activity the agent of which can equally perform on itself or on some other entity, without being pragmatically odd. 
Katarzyna Janic

\subsection{Reflexive-Antipassive Polysemy in the Cariban Family}

Ye'kwana belongs to the Cariban language family and is spoken in Venezuela and Brazil. Significantly, it has the reflexive suffix -öt which, polysemous in nature, performs a range of different functions. Among the many meanings, the reciprocal, anticausative, autocausative, and antipassive tend to be the most common. Example (4) illustrates the reflexive derivation which, similar to the antipassive one in (5), is derived by means of the - ot marker:
(4a) $m \ddot{o} ' d \ddot{o}$
tü $\quad a-j a ' s e-\varnothing=j e$
$m(i)-\ddot{o}$ tö-aanö
DEM.ANIM
INTS 2-niece-POSS=ATRB
2/3-name-PST.IMPF
'The one that is here, you should call her niece.'
(4b) tüwü-:ne kanna kün-öt-ö’tö-aakö
3.SG-INTS probably 3S.PST-REFL-name-PST.IMPF
'It is him who called himself.'
(Cáceres 2010)
$a-j a-d \ddot{u}-j \ddot{u} d \ddot{u}$
uwö i-ökamma-jötü-jai $m a=d \ddot{u} \quad$ naane
2-grandson-POSS-PST.POSS DAT 3O-tell-ITER-HAB 2.COP $=\mathrm{PTC} \quad \mathrm{PTC}$
'You can tell it to your grandson.'

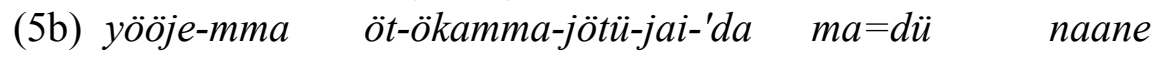
thus-only AP-tell-ITER-HAB-NEG 2.COP=PTC PTC
'You cannot tell [it] only like that (without the picture).'

The sentence in (4a) illustrates a transitive use of the verb - ̈̈'tö- 'name' that occurs with two core arguments, i.e. the pronominal subject and the object. In (4b), the same verbal form is exemplified in its reflexive use. The presence of the reflexive marker -öt affects the syntax and the semantics of the clause, i.e. the object argument is zero-coded and remains coreferential with its subject. Example (5a), on the other hand, illustrates a transitive use of the verb -ökamma 'tell' from which the antipassive one is derived, ex. (5b). Similar to the reflexive, the presence of the -öt marker obligatorily blocks the overt realization of the object in the syntax. Contrary to the reflexive clause, however, the respective argument is not co-referential with its subject.

\subsection{Reflexive-Antipassive Polysemy in the Tacanan Family}

The Tacanan family belongs to the Amerindian languages of South America and is spoken in Bolivia. Among the Tacanan languages, the reflexive-antipassive polysemy is documented in Cavineña.

To derive reflexive and reflexive-like constructions, Cavineña uses the circumfix $k(a)-\ldots-t i$. Similar to Slavic and Cariban languages, the respective 


\section{On the Reflexive - Antipassive Polysemy}

marker presents a number of different functions. Within the scope of meanings, $k(a)-\ldots-t i$ can be used productively to express the reflexive, reciprocal, benefactive, and antipassive meaning (Guillaume 2008). The reflexive and antipassive meanings are exemplified in (6b) and (6c) respectively.

(6a) Señora=ra peta-wa espejo=eke [chapa ushuri=ke] lady $=$ ERG look.at-PFV mirror $=$ PERL dog skinny $=$ LIG 'The lady looked at the skinny dog in the mirror.'

(6b) Señora ka-peta-ti-wa espejo=ju. lady REFL-look.at-REFL-PFV mirror=LOC 'The lady looked at herself in the mirror.'

(6c) Ka-peta-ti-ya =mi-ke? AP-look.AP-IMPF $=$ 2SG-FM 'You are watching [a soccer game]?' (context: this was said in a greeting sense, while I was watching a soccer game.)

(Guillaume 2008:263)

Example (6a) illustrates a transitive use of the verb peta 'look at' from which the reflexive, (6b), and the antipassive, (6c), are derived by means of the circumfix $k(a)-\ldots-t i$. Due to its presence, the zero-coded object argument is either understood as the anaphoric patient of the active clause, ex. (6b), or as a highly referential patient 'soccer game' of the antipassive.

Similar to Cavineña, Ese Ejja also uses the morphologically complex marker, i.e. $x a-\ldots-k i$ that along the reflexive and reflexive-like meanings can also have the antipassive interpretation:

(7a) Eyaya ekwe bakwa jabe-je.

1ERG 1GEN child comb-FUT

'I will comb my child.'

(7b) Epona xa-jabe-ki-ani.

woman REFL-comb-REFL-PRS

'The woman combs herself.'

(8a) Ekwaa motor ishwa-axa-naje

ERG motorboat wait-in.vain-PST

'We waited in vain for the motorboat.'

(8b) Jamaya esea ani-ani xa-ishwa-ki-ani-ani,

so ABS sit-HAB AP-wait-AP-HAB-PRS

'So we usually sit and wait for [a bus].'

(Vuillermet 2010)

Example (7b) expresses a reflexive construction traditionally known in the 
literature under the term 'partitive object reflexive' or 'grooming constructions.' In the given clause, the verbal form jabe 'comb' is derived from the corresponding transitive clause by means of the circumfix $x a-\ldots-k i$. The presence of the latter obligatorily blocks the syntactic realization of the patient argument. Example (8), on the other hand, presents the antipassive use of the circumfix $x a-\ldots-k i$. The sentence (8a) illustrates a transitive use of the verb ishwa 'wait for' from which the antipassive, $(8 \mathrm{~b})$, is derived. Similar to the previous example, the presence of the circumfix $x a-\ldots-k i$ blocks the overt realization of the patient argument. Contrary to the latter, however, the object suppression is subject to different conditions. It is left unrealized on pragmatic grounds. Being semantically implied, the object argument is generic and refers to an unspecified group of vehicles.

\subsection{Reflexive-Antipassive Polysemy in the Manding Family}

The reflexive-antipassive polysemy is also documented in Bambara, a language spoken in western Africa. Similar to Ese Ejja and other languages, Bambara has a morpheme whose main function boils down to reflexive/middle derivation. In certain instances, however, the latter can also perform the antipassive function. Examples (9) and (10) illustrate reflexive and antipassive derivation in Bambara:

(9a) Muso ye denin ko. woman.DEF PFV.POS girl.DEF wash

'The woman has washed the girl.'

(9b)

Muso ye $i \quad k o$.
Woman.DEF ACP.POS REFL wash
'The woman has washed herself.'

(10a) $C \varepsilon \quad$ ye ji min. man.DEF PFV water.DEF drink

'The man has drunk some water.'

(10b) $C \varepsilon \quad y e \quad i \quad \min (j i \quad l a)$.

man.DEF PFV.POS AP drink water.DEF POSTP

'The man has quenched his thirst.'

(Creissels 2006:90)

Sentence (9a) exemplifies a transitive use of the verb ko 'wash.' In (9b), the same verbal form is morphologically marked by the $i$ morpheme which reduces the valency of the verb. Due to its presence, the object argument is zero-coded and becomes coreferential with the subject argument muso 'mother.' In (10), the same morpheme performs the antipassive function. Like in the previous example, the presence of the $i$ morpheme affects the syntactic properties of the construction in a way that it becomes syntactically intransitive. Contrary to the previous example, 


\section{On the Reflexive - Antipassive Polysemy}

however, the object argument is not co-referential with the subject but is demoted to the oblique position. As a result of its syntactic demotion to peripheral status, the object argument loses the properties of a core argument. Significantly, the transitive/antipassive alternation does not affect the semantic roles of the core arguments. Both the subject and object arguments in (10b) assign the same semantic role, i.e. the agent and the patient role as their counterparts in the transitive clause (10a).

\subsection{Reflexive-Antipassive Polysemy in the South Caucasian Family}

The South Caucasian family is another class of languages in which the reflexiveantipassive polysemy is often encountered. Among languages subsumed under the South Caucasian family, Laz in particular is known for the multifunctional use of its reflexive marker $i$-.

Similar to the previous languages, Laz uses a reflexive marker to express the antipassive meaning. Significantly, the respective morpheme expresses also a number of other meanings which are usually considered to carry the meanings of middle forms. Alongside the reflexive proper and the antipassive, $i$ - can be used productively to express the autocausative, auto-benefactive, anticausative, and facultative meaning. Typologically-oriented studies usually refer to this type of marker as a middle marker. Interestingly in Laz, the $i$ - suffix can also be used to derive non-middle constructions such as passives and impersonals.

Example (11) illustrates a ditransitive use of the verb -gur- 'learn'; from which the reflexive dative, (12), and the antipassive, (13), are derived:

$$
\begin{array}{lllll}
\text { padisahi-k } & \text { jur } & c^{\prime} \text { 'ut'al-epe-s } & \text { zanaxat } & \text { d-o-gur-am-t'u } \\
\text { sultan-ERG } & \text { two } & \text { little-PL-DAT } & \text { profession } & \text { PREV-VAL1-learn-STH- } \\
& & & & \text { IMPF.I3SG }
\end{array}
$$

'Sultan taught two boys a profession.'

(12) bere-k ir sey ko-d-i-gur-u-dort'un child-ERG every thing PV-PV-REFL-learn-I3SG-PQP

'The young child has learnt everything.'

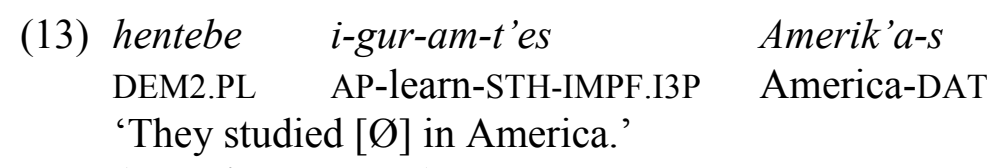

(Lacroix 2009:467)

In (12), the verbal stem -gur- is marked by the morpheme $i$-. Due to its presence, the subject argument berek 'child' is co-referential with the dative. The presence of the reflexive marker does not affect the syntactic properties of the subject. 
Katarzyna Janic

Similar to the corresponding ditransitive clause, the berek argument is still in the ergative case. In (13), the same verbal form -gur-is also derived by means of the $i$ - morpheme, however, in this example it performs the antipassive function. Due to its presence, the construction in question takes on the formal characteristics of the intransitive clause, i.e. the subject argument that in the ditransitive clause was ergative is now in the absolutive and the object argument that was eliminated from the syntax of the clause is interpreted as the non-referring and non-topical patient meaning 'something.'

\subsection{Reflexive-Antipassive Polysemy in the Pama-Nyungan Family}

The Australian Pama-Nyungan family is significant for the present discussion in two respects. First, it is not genetically related to the Indo-European languages. Second, apart from regular antipassives, it also developed the so-called structural antipassive (Cooreman 1993). The latter, strongly related to the ergative system, is used to bypass certain syntactic constraints imposed on the subject of transitive constructions.

Although the Pama-Nyungan family is not genetically related to IndoEuropean languages, like the latter, it exemplifies instances of the reflexiveantipassive polysemy. The following example comes from Warrungu, the PamaNyungan family and illustrates the reflexive and antipassive derivation, respectively:

(14a) Gaya-nggu bama-Ø giba-n.

father-ERG man-ACC shave-NONFUT

'Father shaved a man.'

(14b) Gaya-Ø giba-gali-Ø.

father-NOM shave-REFL-NONFUT

'Father shaved himself.'

(15) Bama-Ø jurba-nggu bangga-gali-n.

man-NOM white.ochre-ERG paint-AP-NONFUT

'The man is painting [someone else] with white ochre.'

(Tsunoda 2006:305)

In Warrungu, the suffix -gali attached to the verbal root is used mainly to express reflexive and reflexive-like actions, (14b). In certain instances, however, it can also perform the antipassive function, (15). Data on Warrungu reveal, however, few instances in which -gali expresses the antipassive reading. In most cases, its function boils down to the reflexive derivation. Although the antipassive meaning may seem accidental for the Warrungu reflexive suffix, it is not accidental typologically, as it is concomitant with the reflexive meaning in a wide range of 


\section{On the Reflexive - Antipassive Polysemy}

languages.

Contrary to Warrungu, Yidiny developed two types of antipassive constructions, i.e. the regular one (16), and the one used for syntactic purposes:

\section{(16) yayu bae mbi-:dji-nju}

1SG cover-REFL/AP-PST

'I covered myself,' or 'I covered [someone/something].'

(Terrill 1997:83)

Example (16) illustrates the reflexive-antipassive polysemy actualized on the same verbal form bambi 'cover.' Consequently, a given derivative is ambiguous, vacillating between two meanings. In such a case, a proper interpretation depends on the relevant pragmatic context. Note that the presence of the -:dji suffix triggers the verbal valency. Due to its presence, the object argument is removed from the syntax, though not from the semantics of the clause. Depending on the given context, a zero-coded argument may be either interpreted as the anaphoric patient of the active clause or as a non-referring patient of the antipassive.

In Yidiny, -:dji suffix can also be used to derive structural antipassives. In comparison to antipassives used for semantic/pragmatic reasons (16), the latter is used to feed syntactic pivots. It often has various functions and syntactic forms. In Yidiny a structural antipassive, (19), is used to coordinate two clauses:

(17) yinjdju:y bama-:l mayi djula:l these-ERG people-ERG vegetables.ABS dig.up 'These people dug up vegetables.'

$\begin{array}{llll}\text { yayu } \quad \text { yigu bama } & \text { bandji:li-nju } \\ \text { 1SG.NOM these people.ABS } & \text { find.PST } \\ \text { 'I went and found these people.' } & \end{array}$
yayu yigu bama
bandji:li-nju
mayi-:
djula-:dji-nju:n
SG.NOM these people.ABS find.PST
veg-LOC dig.up-AP-PST

'I went and found these people digging up vegetables.'

(Terrill 1997:85)

Examples (17) and (18) illustrate transitive clauses with the common argument 'these people.' To coordinate the respective constructions, referentially identical arguments must be both absolutive across coordinated clause boundaries. When the respective argument is not syntactically absolutive, a special construction, i.e. the structural antipassive, intervenes to bypass this constraint, as in (19). In other words, in (19), the ergative agent of the first clause cannot be deleted under coreferentiality with the absolutive object of the second clause, unless it is syntacti- 
cally absolutive. Thus, in order to delete a respective argument, it must be first marked by the same case as the object argument of the second clause. To achieve this goal, the structural antipassive is used.

\subsection{Reflexive-Antipassive Polysemy in the Chukotko-Kamchatkan Family}

The Chukotko-Kamchatkan family is another example of languages in which the reflexive-antipassive polysemy is frequently attested. In Chukchi, a language spoken in northeastern Siberia, antipassive constructions are derived by means of the prefix ena-/ine- or by the suffix -tku/-tko. The latter are of a particular interest here because of their typologically distinctive polyfunctionality.

Apart from the antipassive function, they also allow the reflexive, reciprocal, and the anticausative interpretation. Contrary to the previous languages, however, the primary function of the respective suffixes is not related to reflexivity but to reciprocity, (Nedjalkov 2006). The reciprocal and antipassive derivations are illustrated in (20) and (21) respectively:

they.ABS man-as kiss-ə-RECIP-AOR.3PL

'They kiss each other as men do.' (situation: 'they kissed once only')

(21a) ' $3 t t^{2}-e$ juu-nin

'The dog bit him.'

(21b) ?tt ${ }^{2}-\partial n$ no-j gu-tku-qin

'The dog bites [people].'

(Nedjalkov 2006:222)

Interestingly, in terms of referential properties of the patient argument in (21), the antipassive derivation resembles that of Slavic languages illustrated in (2). In both examples, a zero-coded object argument may refer to a definite or unspecified individual or to a loosely specified group of individuals and always displays [+human] properties.

\section{Givón's Diachronic Scenario}

After having investigated the reflexive-antipassive polysemy in a range of various languages, we can now turn to the chief question of this paper, namely: on what grounds did the antipassive develop from reflexivity? To show that antipassives extended from reflexivity on historical grounds, I will base my arguments on the diachronic properties of the reflexive marker in Indo-European languages.

The historical explanation of the shared morphology will be particularly 


\section{On the Reflexive - Antipassive Polysemy}

accounted for on the basis of Givón's functional approach (2001:94) in which he claimed that "detransitive constructions, most conspicuously the passive, commonly arise via re-grammaticalization of some functionally related construction" and that "functional extension of syntactic constructions is driven primarily by functional similarity." In other words, I will argue that similar to the passive, the antipassive developed diachronically from the de-transitive, i.e. the reflexive construction through functional extension, and that functional extension was primarily initiated by functional similarity. Thus, if we manage to find functional similarity between the source and the target construction, then it will be legitimate to claim that the antipassive developed from reflexivity through the functional extension, regardless of the language system.

\subsection{Functional Properties of Reflexive and Antipassive}

The partial functional overlap between reflexives and antipassives pertains mainly to semantic and/or pragmatic dimensions. Both types of construction deviate from their corresponding transitive counterpart on semantic grounds, i.e. they are said to express a semantically less transitive action. Building on the transitivity parameters of Hopper and Thompson (1980), the constructions in question are ranked lower on the semantic transitive scale in three respects.

First, they are characterized by a decreased agentivity of the agent/subject argument. In the reflexive construction the argument in question is less agentive due to the coreference requirement, whereas in the antipassive one a decreased agentivity results from the fact that the agent is often presented not as being voluntarily engaged in a particular type of activity but rather as having a certain predisposition to perform it. This is due to the fact that the antipassive derivation often affects the meaning of the verb that subsequently denotes a habitual action. Consequently, the latter may become a permanent characteristic of the subject.

A lower degree of transitivity of reflexives and antipassives is also visible in terms of a type of activity expressed by the verb. Both constructions express the action that is semantically less transitive. As for reflexives, their events are in general semantically less transitive because of the number of participants involved in the action. A semantic definition of a transitive event requires two highly distinct participants of different semantic properties, whereas in reflexives, due to the coreference condition, only one participant is involved. Antipassives, on the other hand, differ from the corresponding transitive event in that they often denote action that is iterative, habitual, and cognitively non-salient.

Finally, reflexives much like antipassives have a non-distinct object argument whose absence is left unmarked in the syntax of the clause. Consequently, both constructions are structurally identical, i.e. the object argument is removed and a verbal form is marked with the special morphology. As such the respective constructions are syntactically intransitive. The only difference pertains to the 
conditions under which the argument at issue is omitted. In reflexives, the object argument is removed because of the coreference condition, whereas in antipassives because of its irrelevance to the discourse context. As a result, both constructions differ in terms of the interpretation of the zero-out object. Depending on the given example, an object may be considered to be either the anaphoric patient of the active clause or a non-referring, non-topical patient of the antipassive. Thus, for both reflexives and antipassives, there is a functional similarity between the two different uses of the zero argument: "unexpressed information in general is prompted by two major cognitive-functional factors: (a) predictability and (b) irrelevance" (cf. Givón 2007:22 for the passive).

\subsection{From Reflexive to Antipassive: a Possible Scenario}

Building on the functional similarity between reflexives and antipassives, we can now proceed to a possible diachronic scenario of the given construction.

In the first stage of the development, speakers use the reflexive constructions to code a reflexive event. Consequently, the action denoted by a verb becomes semantically less transitive, the agent less agentive, and the patient, being coreferential with the agent, pragmatically less focused, less salient, and functioning as a non-distinct argument. Such reflexive events are syntactically coded by the zerocoded object and the reflexive marker on the verb.

Now, being confronted with a similar pragmatic situation, but in which the patient was not coreferential with the agent, individual speakers may have extended the use of the old construction to code this new, though very similar, situation. In other words, they used the same syntactic structure in a new discourse context in which the patient was not coreferential with the agent; however, all other functions of the situation remain unchanged. Thus, the same construction whose primary function was restrained to reflexivity started to be used to perform two similar but not identical functions, i.e. the old (reflexive) and the new (antipassive) with the latter viewed as the extension of the former (cf. Terrill 1997 for Australian languages). Consequently, a respective construction was sometimes ambiguous, vacillating between two meanings.

Now, depending on the grammatical system of the given language, either a complete separation of the two functions into different constructions took place (cf. section 2.6 for Australian languages), or the structural adjustment did not occur and a respective construction remained ambiguous, performing two functions (cf. section 2.1 for Slavic languages).

\subsection{Antipassive in Early vs. Late Stage of Grammaticalization}

Let us now turn to the last issue related to the reflexive-antipassive polysemy, i.e. the degree of grammaticalization of a given construction. Estimating to what 


\section{On the Reflexive - Antipassive Polysemy}

extent reflexives underwent a grammaticalization process in the respective languages is crucial for the present discussion in two respects. First, it helps us to determine decisive factors that contribute to the grammaticalization process. Second, it shows how far a given construction has progressed along the reflexiveantipassive road. In other words, it allows us to assess whether a respective construction evolved into a canonical type or whether it is still in the evolutionary process characterized by functional ambiguity.

Building on Givón's functional approach, we can presuppose that what gave rise to the functional extension of reflexives is, undoubtedly, the functional similarity between the source and the target construction. Indeed, functional similarity is considered to be one of the defining factors, if not the only one, in the diachronic evolution of the source construction. In addition, it is said that the early stage of development during the grammaticalization process is characterized by functional ambiguity. This is because the same syntactic structure tends to perform two similar, however not identical, functions: the old and the new one. As such, a given construction is ambiguous. This is exactly what is observed for instance in Slavic in (3) or in Pama-Nyungan languages in (16), where the same construction was used to express both reflexive and antipassive functions.

Note that functional ambiguity is a distinguishing feature of early stages of any grammaticalization process. This is because functional re-analysis that usually takes place instantaneously as a spontaneous speech act of individual speakers is considered to be the earliest step in the diachronic extension. Once the use of the source construction in the new pragmatic context becomes a regular speech act, the syntactic re-analysis or adjustment of the given construction eventually takes place. The syntactic re-analysis is expected to occur at a more advanced level of the grammaticalization process and is reflected by the change in the syntactic form of the given construction (Givón 2007). This is what was observed in Yidiny, an Australian Pama-Nyungan language, in (19).

Building on different levels of the grammaticalization process, it is clear now that the reflexive-to-antipassive extension is not at the same stage of development in all given languages. Contrary to Australian languages in which the evolution was a three-step process terminating with syntactic re-analysis of the reflexive construction, a diachronic change of reflexives in the remaining languages was only a two-step process resulting in ambiguous patterns. In this case, depending on the lexical meaning of the verb and/or pragmatic context, either both interpretations were equally acceptable, ex. (3) or ex. (16), or the antipassive reading was more or less strongly preferred, ex. (2). This means that contrary to Australian languages in which the reflexive-to-antipassive extension is in a late stage of development, in the all remaining languages antipassives are still at the beginning of grammaticalization characterized by functional ambiguity. 


\section{$4 \quad$ Concluding Remarks}

The aim of this paper was to show that the reflexive-antipassive pattern in respective languages is by no means homophonous in nature but rather it results from the diachronic properties of the reflexive marker. To show that the morphological overlap between reflexives and antipassives is historically grounded, regardless of the language system, I based my arguments on Givón's functional approach which is relevant for the present discussion in two main respects. First, it helps us to determine key factors that contribute to the grammaticalization process of the given construction. Second, it allows us to estimate the degree of grammaticalization.

In relation to this issue, we have seen that only in Australian languages have antipassives completely undergone the grammaticalization process. This means that only for these languages has the reflexive covered the distance from the postulated source construction to what may be interpreted as the target construction. In the other languages examined the antipassive is still in its early stage of development, characterised by functional similarity.

\section{Abbreviations}

$\begin{array}{llllll}\text { ABS } & \text { Absolutive } & \text { GEN } & \text { Genitive } & \text { POS } & \text { Positive } \\ \text { ACC } & \text { Accusative } & \text { HAB } & \text { Habitual } & \text { POSS } & \text { Possessive } \\ \text { ANIM } & \text { Animate } & \text { IMPF } & \text { Imperfective } & \text { POSTP } & \text { Postposition } \\ \text { AOR } & \text { Aorist } & \text { INTS } & \text { Intensive } & \text { PRS } & \text { Present } \\ \text { AP } & \text { Antipassive } & \text { ITER } & \text { Iterative } & \text { PST } & \text { Past } \\ \text { ATRB } & \text { Attributive } & \text { LIG } & \text { Ligature } & \text { PTC } & \text { Particle } \\ \text { COP } & \text { Copula } & \text { LOC } & \text { Locative } & \text { PQP } & \text { Pluperfect } \\ \text { DAT } & \text { Dative } & \text { NEG } & \text { Negation } & \text { PREV } & \text { Preverb } \\ \text { DEF } & \text { Definite } & \text { NONFUT } & \text { Non-future } & \text { REFL } & \text { Reflexive } \\ \text { DEM } & \text { Demonstrative } & \text { NOM } & \text { Nominative } & \text { RECIP } & \text { Reciprocal } \\ \text { ERG } & \text { Ergative } & \text { PFV } & \text { Perfective } & \text { SG } & \text { Singular } \\ \text { FM } & \text { Formative } & \text { PERL } & \text { Perlative } & \text { STH } & \text { Suffix } \\ \text { FUT } & \text { Future } & \text { PL } & \text { Plural } & \text { VAL } & \text { Valency }\end{array}$

\section{References}

Cáceres, Natalia. 2010. Détransitivisation et voix moyenne en ye'kwana. Atelier Morphosyntaxe, DDL, 19 March.

Cooreman, Ann. 1993. A Functional Typology of Antipassive. In B. Fox and P. J. Hopper, eds., Voice: Form and Function, 49-87, Amsterdam: John Benjamins Publishing Company. 
On the Reflexive - Antipassive Polysemy

Creissels, Denis. 2006. Syntaxe générale, une introduction typologique. Paris: Hermès.

Dixon, Robert M. W. 1979. Ergativity. Language 55:59-138.

Givón, Talmy. 2001. Syntax: An Introduction. II. Amsterdam/Philadelphia: John Benjamins Publishing.

Givón, Talmy. 2007. On the Relational Properties of Passive Clauses: A Diachronic Perspective. In Z. E. Fernández, S. Wichmann, C. Chamoreau, and A. A. González, eds., Studies in Voice and Transitivity, 19-32, München: Lincom Europa.

Geniušienè, Emma. 1987. The Typology of Reflexives. Berlin: Mouton de Gruyter.

Guillaume, Antoine. 2008. A Grammar of Cavineña. Berlin: Mouton de Gruyter.

Heath, Jeffrey. 1976. Antipassivization: A Functional Typology. Proceedings of the Second Annual Meeting of the Berkeley Linguistics Society, 202-211.

Hopper, Paul and Sandra A. Thompson. 1980. Transitivity in grammar discourse. Language 56: 251-299.

Lacroix, René. 2009. Description du dialecte laze d'Arhavi, (caucasique du sud, Turquie): Grammaire et textes. Ph.D. thesis, Université Lumière Lyon 2.

Nedjalkov, Vladimir P. 2006. Chukchi Reciprocals. In Tasaku Tsunoda and Taro Kageyama, eds., Voice and Grammatical Relations, 217-246, Amsterdam/Philadelphia: John Benjamins Publishing.

Nedjalkov, Vladimir P. 2007. Polysemy of Reciprocal markers. In Vladimir P. Nedjalkov, ed., Reciprocal Constructions, 231-334, Amsterdam/Philadelphia: John Benjamins Publishing.

Polinsky, Maria. 2005. Antipassive Constructions. In Martin Haspelmath, Matthew S. Dryer, David Gill, and Bernard Comrie, eds., The World Atlas Of Language Structure, 438-439, Oxford: Oxford University Press.

Terrill, Angela. 1997. The Development of Antipassive Constructions in Australian Languages. Australian Journal of Linguistics 17(1):71-88. 


\section{Katarzyna Janic}

Vuillermet, Marine. 2010. Le moyen en ese ejja. Atelier de morphosyntaxe, DDL, 9 April.

Katarzyna Janic

Laboratoire Dynamique Du Langage (UMR 5596)

Institut des Sciences de l'Homme

14 avenue Berthelot

69363 Lyon, France

Katarzyna.Janic@univ-lyon2.fr 